\title{
Transplantation and Identity: A dangerous split?
}

\author{
Carosella E.D.*, and Pradeu T. ${ }^{\dagger}$
}

Final version published in The Lancet (2006)

Since the first live kidney transplant in 1952, and the first heart transplant in 1967, the practice of transplantation has provoked many ethical questions, questions which relate more to the donor than the patient (recipient). Answers to these questions vary, depending on countries and cultures; practices can range from donation (cadaveric or live) to organ trade to a strict transplantation ban. In France and the U.K., a deceased person is considered a potential organ donor unless the family explicitly refuses to allow the donation. The fact that the final decision rests with the family indicates that transplantation raises cultural issues, including the respect of individuality and kinship. In this example, the identity of the individual goes beyond himself and extends to the whole family where the ethical and medical decision of organ donation is concerned. Until recently, when transplant procedures were carried out only in life threatening cases, the ethical concerns of the donor were more acute than those of the recipient, except in cases where receiving an organ was banned on religious or cultural grounds. Furthermore, transplanted organs were internal and non-visible, and as such the identity of the organ recipient was not deeply put into question, even if sometimes he could not help imagining the donor's life and character.

A major change occured with the advent of visible and non vital organ transplants: in particular the hand (France 1998 $)$ and the face (France 2005²). These transplantations raise new ethical issues, both for the donor and the recipient. On the donor's side, ethical issues are focused on the family circle, who must face the continuing identity of a loved one, for instance when the hand (or, even more significantly, the face) of a deceased relative comes to life again on someone else. Although the donation of visible organs is scarce, the subject is given much publicity in the media; anonymity of the donor and of the recipient can hardly be preserved making these ethical issues all the more pressing.

\footnotetext{
* Service de Recherches en Hémato-Immunologie, Commissariat à l'Énergie Atomique CEA-DRM-DSV, Hôpital Saint-Louis, Paris, France.

${ }^{\dagger}$ Institut d'Histoire et de Philosophie des Sciences et des Techniques (IHPST), Department of Philosophy, Paris1-Sorbonne University, Paris, France.
} 
The transplantation of visible organs provokes more serious questions for the recipient. Visible organs are direct expressions of an individual's identity and they are strongly involved in relationships with other people and in the image conveyed by the individual. Hands embody a very important means of communication, and they retain the past and personality of the individual. The face expresses even more directly the identity of the person. For a recipient, the every day encounter with a visible transplant can be difficult, since it implies accepting the presence of another person, and a modified expression of the recipient's personality. In the case of a visible transplant, the donated organ exercises not only an organic function, but also the expression of this function. Thus it contributes to the formation of the image that the individual has of himself, to the image others have of him, and above all to the image that he believes the others have of him (social mirror image).

With the transplant of a visible organ a deep identity split occurs, because the image of oneself is modified dramatically. Even if functionality is given to the grafted organ, the recipient still has to reconstruct the expression of this new organ, which is both himself and different from himself. In some cases, the transplantation is essential, particularly when the patient's life is threatened by a state of depression ${ }^{3}$. Therefore, both the living conditions of the patient and his capacity to rebuild his identity must be taken into account. For instance, New Zealander Clint Hallam received a hand graft in 1998, but his hand, which he started to consider as "other" ("foreign"), became unbearable for him; he stopped taking immunosuppressive drugs, a rejection occurred, and eventually he asked that his hand be amputated $^{4}$. Every graft of a visible organ leads to an identity split, the consequences of which can be very serious if the recipient does not succeed in rebuilding the expression as well as the functionality of the organ. Thus a transplantation can be considered successful if it assures not only the function of the organ, but also the rebuilding of the recipient's identity.

This rebuilding work, though difficult, can be fruitful, because identity is precisely characterized by a continuous evolution. The graft of a visible organ can lead to a full expression of one's identity, making the individual aware that to be oneself is to change constantly, and to accept oneself as changing.

In conclusion, it is crucial that ethical debates be opened nowadays to specific questions concerning the projection of the donor's identity, and the rebuilding of the recipient's identity. Grafts of visible organs can be considered successful when they enable both good functionality and a rebuilding of the identity of the person, based on a renewed and wellaccepted "oneself". 


\section{Acknowledgements}

We thank Hannah-Louise Clark for her help.

${ }^{1}$ Dubernard J-M, Owen E, Herzberg G, Lanzetta M, Martin X, Kapila H, Dawahra M, Hakim NS. Human hand allograft: report on first 6 months. Lancet 1999; 353: 1315-1320.

${ }^{2}$ The first facial transplant. Lancet 2005; 366: 1984.

${ }^{3}$ Hovius S.E.R. Hand Transplantation - An Opinion. The Journal of Hand Surgery 2001; 26B: 519-520.

${ }^{4}$ Meyer V.E. Editorial: Hand Transplantation. The Journal of Hand Surgery 2001; 26B: 509-510. 